\title{
ÉTUDE PRÉLIMINAIRE DE QUELQUES QUALITÉS DES CIRES GAUFRÉES PRÉSENTÉES AUX ABEILLES
}

R. DARCHEN

Station de Recherches Apicoles Bure-sur-Yvette
$P+A$

et

Station expérimentale d'Apiculture Montfavet

Étant donné le nombre sans cesse croissant des différentes espèces de cires gaufrées sur le marché apicole, il était intéressant de rechercher quelles étaient les qualités essentielles à développer pour les amorces des rayons.

Dans ce premier travail nous en avons étudié trois en excluant les autres.

Il s'agit d'obtenir :

$I^{\circ}$ Une bonne acceptation des matériaux par les abeilles.

$2^{\circ}$ Des constructions régulières à l'intérieur des ruches à cadres mobiles.

$3^{\circ}$ Un couvain bien compact et bien réparti sur les rayons.

\section{I. - Acceptation des cires par les abeilles.}

Dans 9 ruches Dadant Blatt Io cadres, entièrement neuves, nous avons mis en compétition six espèces de cire. L,es ruches, ayant même orientation et même revêtement, ont été peuplées avec neuf essaims d'abeilles noires de même provenance, enruchés le même jour et par la même technique. Nous avons nourri ces colonies de manière égale et abondante, les abeilles ayant accès au sirop tout le long des têtes de cadres et non en un point central qui pourrait fausser les expériences. I.es six espèces de cires se répartissent suivant le tableau $\mathrm{I}$.

A l'intérieur des ruches, les cadres ont été groupés par 3 ou 4 d'une même espèce et dans un ordre différent pour chaque ruche afin d'éviter un effet de position possible. On trouve dans chaque colonie 3 espèces de cires différentes en allant de gauche à droite, mais jamais dans le même ordre.

Le tableau II suivant représente dans le détail la position de ces cires.

Les premières constructions sont seules intéressantes : en effet, suivant la position des premières cires étirées, les feuilles voisines sont adoptées ensuite automatiquement dès l'extension de la grappe d'abeilles. 
TABLEAC I

\begin{tabular}{|c|c|c|c|}
\hline dortire & $\begin{array}{c}\text { Conposition approximative } \\
\text { des rires gaufrés }\end{array}$ & $\begin{array}{l}\text { Nombre de } \\
\text { cadres épuilines } \\
\text { au tontal }\end{array}$ & $\begin{array}{l}\text { Apredlation } \\
\text { référence }\end{array}$ \\
\hline 1 & Cire ommonerciale diabeilles & 27 & $A$ \\
\hline 2 & 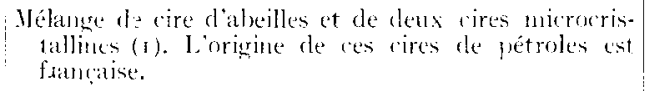 & 18 & B \\
\hline 3 & (Cire d'abeilles, extraite de rabons latis far l'atctome. & 16 & $c^{\circ}$ \\
\hline i & 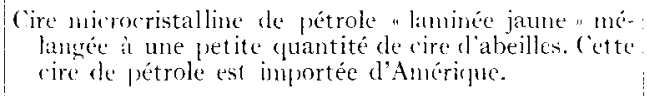 & 6 & I) \\
\hline 5 & 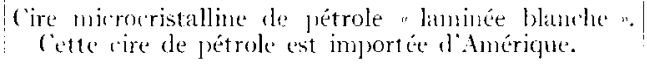 & $i$ & $\mathrm{I}$ \\
\hline 6 & 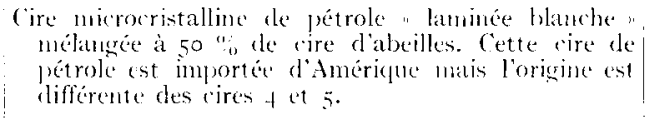 & 16 & Ii \\
\hline
\end{tabular}

(1) les cires mirorristallines (muployées sont toutes des cires de petrole it bas point de fusion

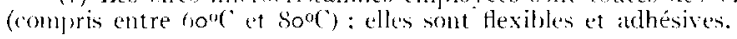

'TABI.EAT II

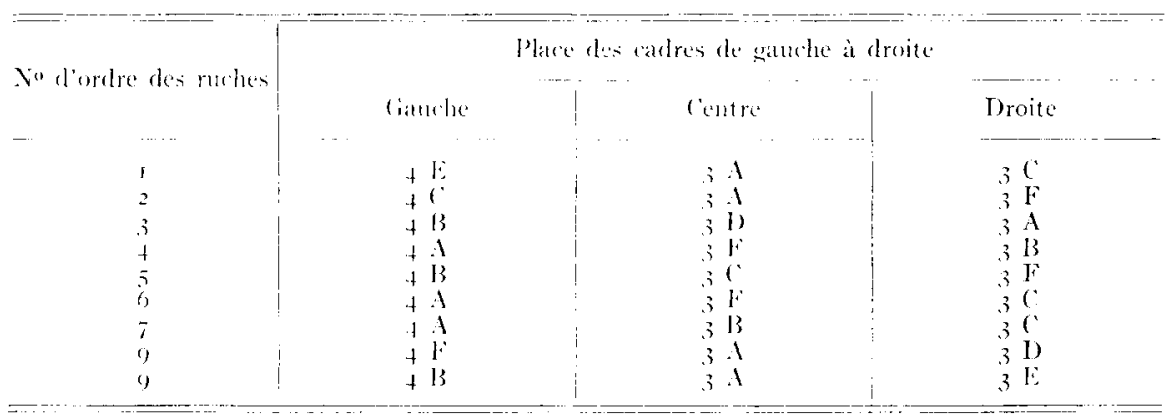

(Noter que si les premières constructions sont an centre car on trouve alors deux possibilités à gauche et à droite).

Quatre jours après l'enruchement, on observe les résultats résumés daus le tableau III.

Ces résultats nous permettent d'établir le tableau IV suivant qui indique les cires gaufrées les mieux acceptées par les essaims.

in conclusion, on peut aftirmer que:

I $^{\circ}$ I,es abeilles acceptent des cires et des matières très diverses (notamment des cires minérales) au début de leurs constructions.

$2^{\circ}$ Elles préfèrent, semble-t-il, les feuilles gaufrées dans lesquelles entre une certaine proportion de cire d'abeilles, qui donne au mélange nue assez grande malléabilité (C et 1$)$ ).

$3^{\circ}$ Elles ne semblent pas préférer la cire d'abeilles à certaines autres cires (A). 


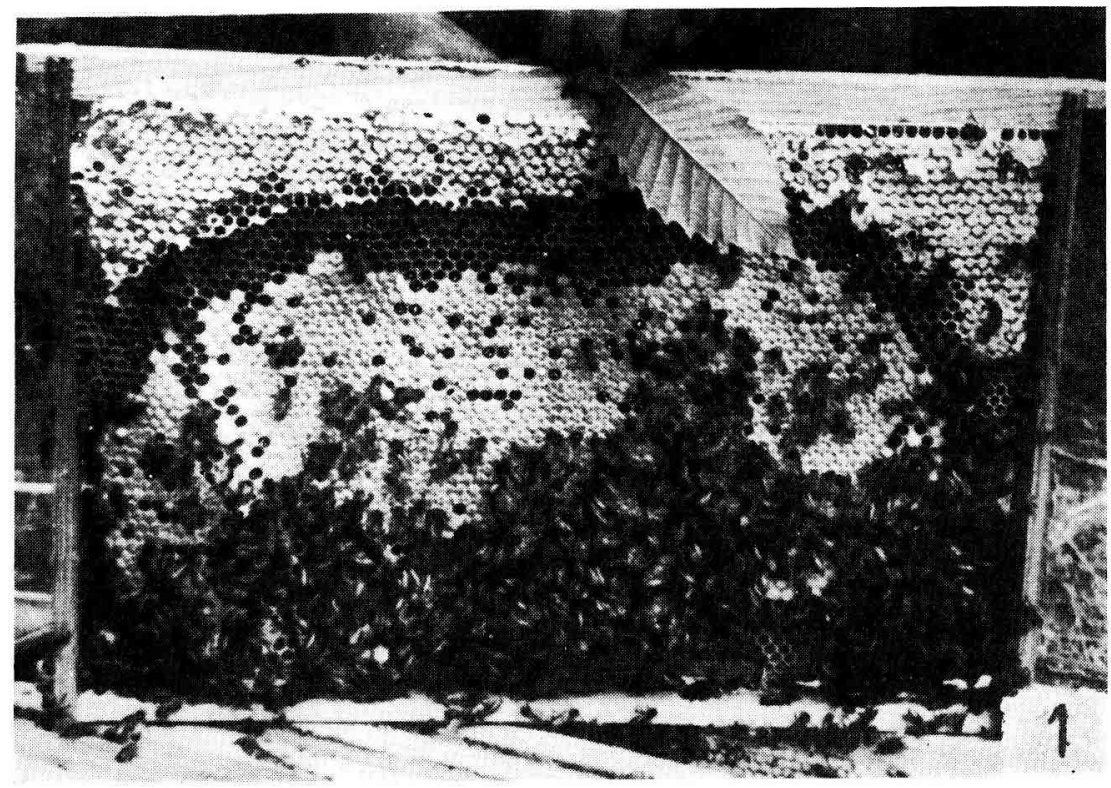

FIG. 1.

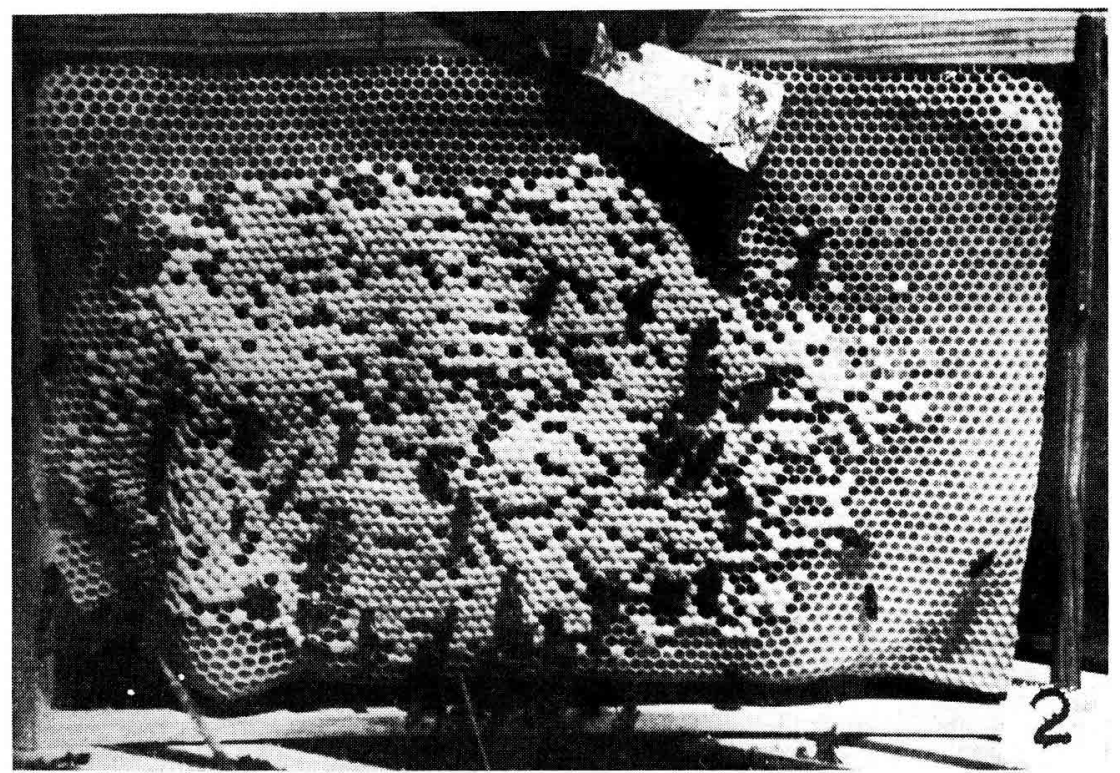



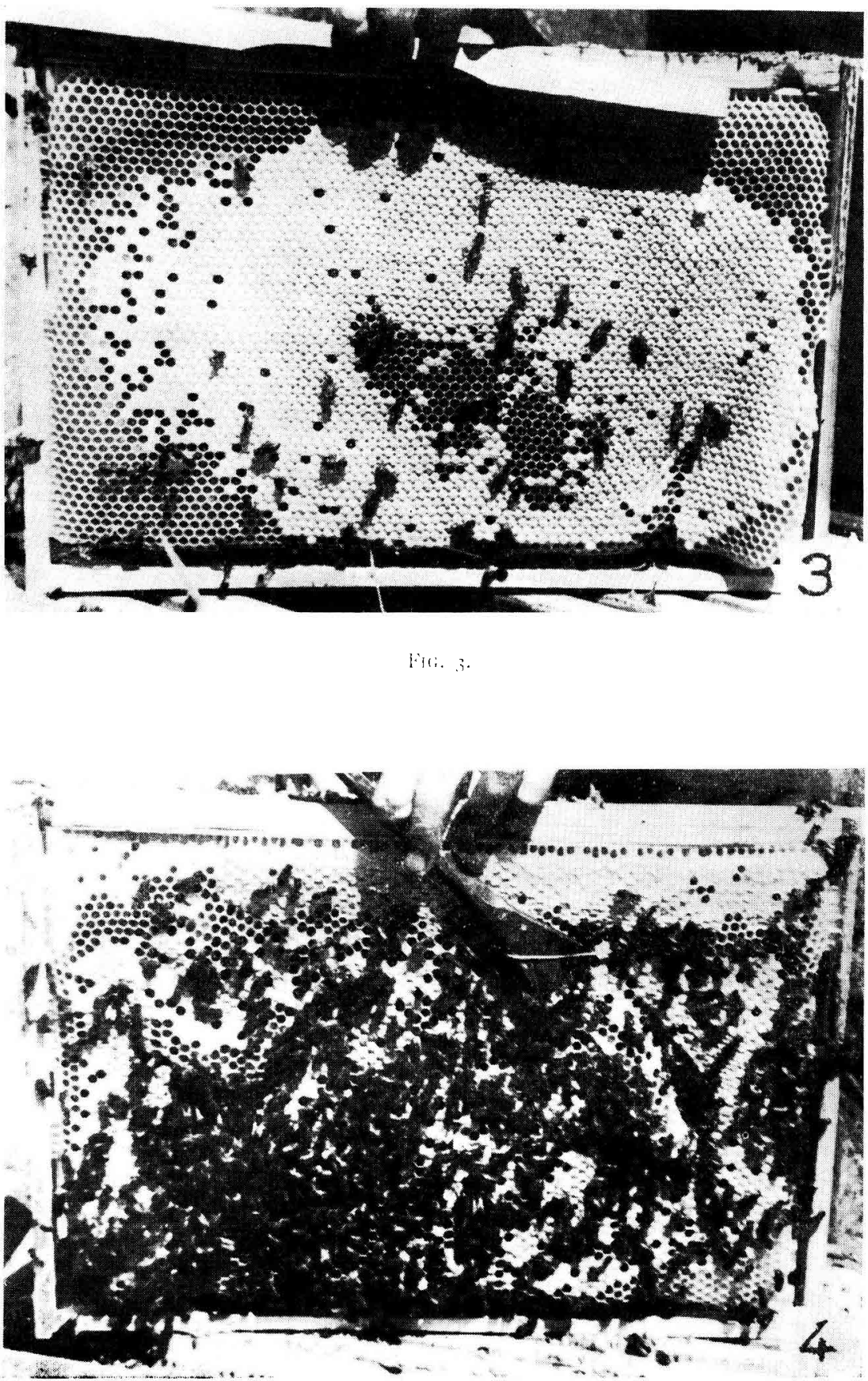
TABLALA III

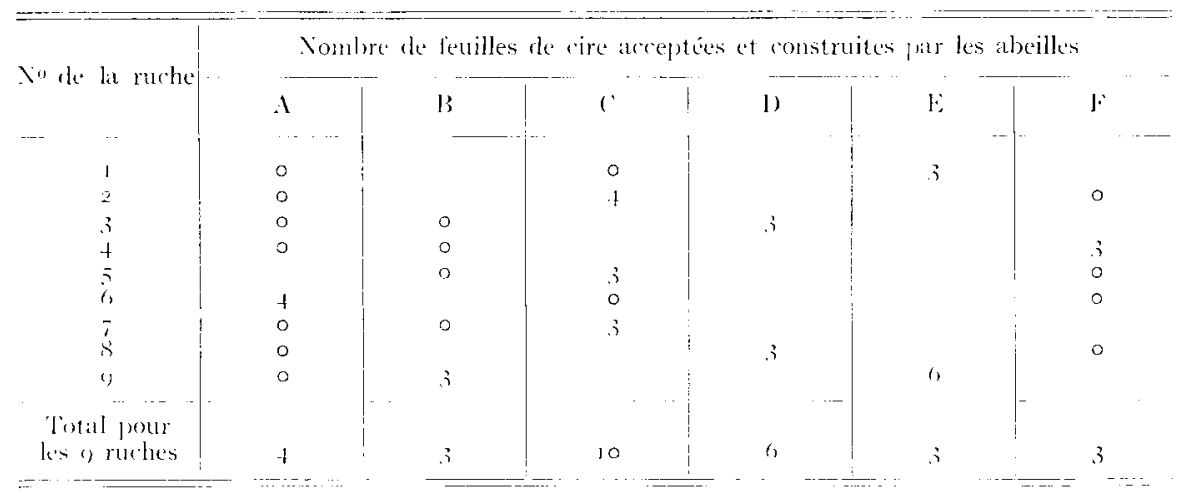

TABITLAU IV
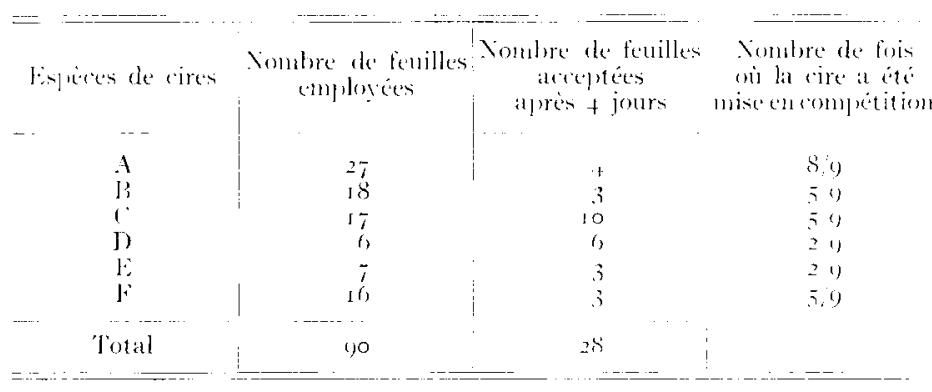
ail la rire a equ miscencomputition!

Combre de fois

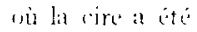
areptice

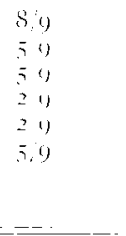

\section{II. - Mégularité des constructions.}

Ia qualité d'une cire gaufrée, en ce qui concerne la régularité des constructions après étirage, n'a pas été l'objet jusqu'ici d'une grande attention. Il suffit bien souvent qu'une cire soit bien acceptée par les abeilles pour être introduite sur l' marché apicole. Cependant de graves ennuis penvent survenir; avec certaines cires trop malléables des rayons s'écroulent à l'intérieur de la ruche, la feuille de cire se tord sous l'action des fortes chaleurs arant ou pendant la construction, les abeilles anorcent de nombreuses constructions parasites.

Le tableau $V$ résume les résultats obtenus avec les différentes catégories de cire que nous avons testées.

I.es photographies complètent le tableau précédent.

En résunié, la régularité des constructions nécessite :

I $^{0}$ La présence d'une certaine proportion de cire d'abeilles dans les feuilles gaufrées.

$2^{\text {o }}$ Un certain coefficient de malléabilité qui permette une bonne acceptation des cires et évite cependant les gondolements et les effondrements. 


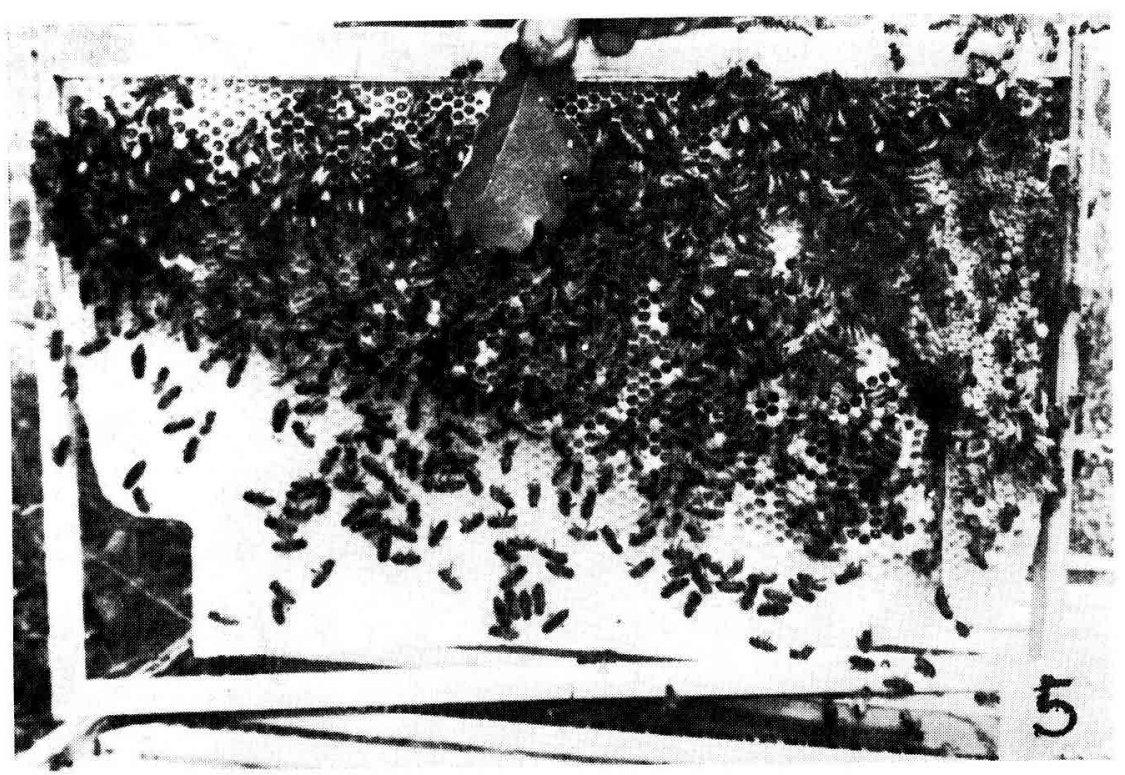

Fir. 5

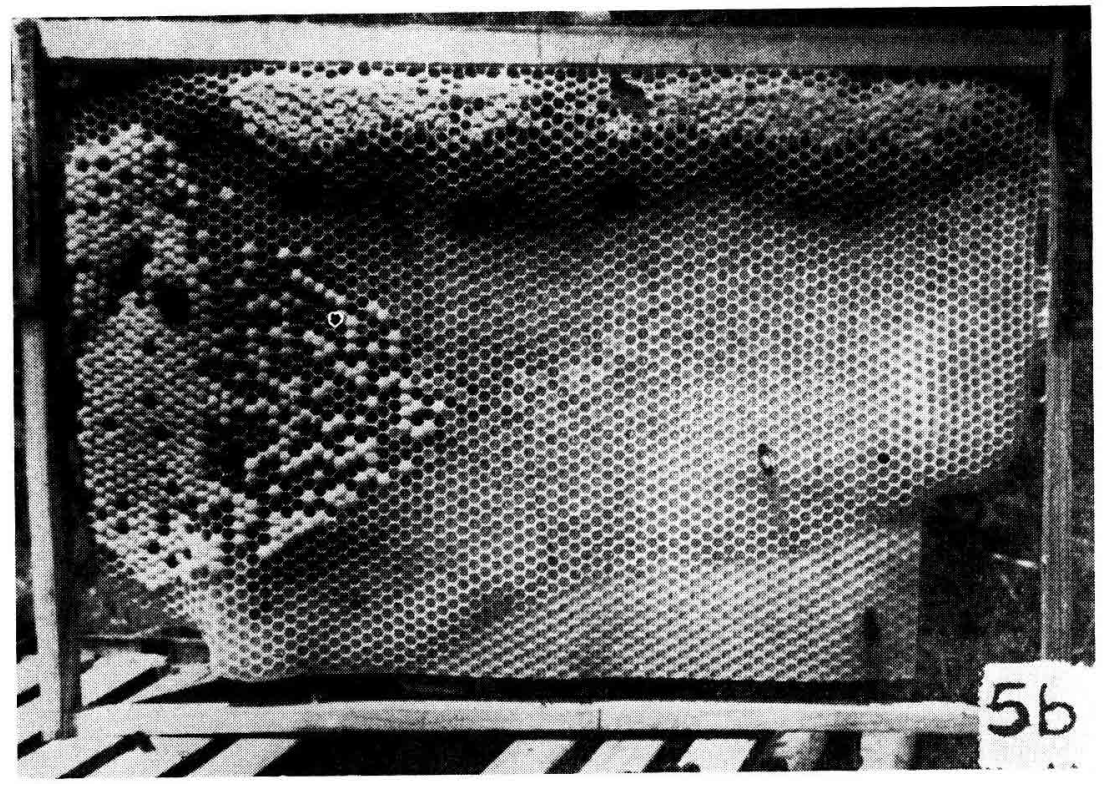

FIG. $5 \%$ 


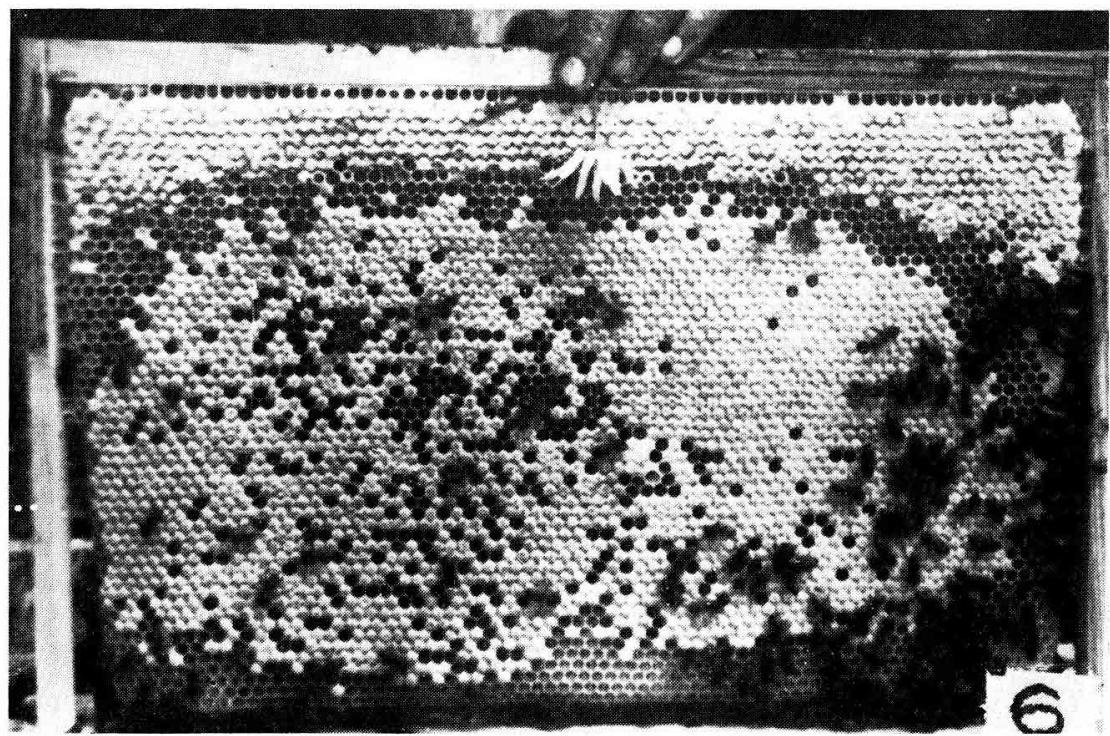

IFIs. \%.

TABLEAT Y'

\begin{tabular}{|c|c|}
\hline $\begin{array}{c}\text { Categrorie de cire } \\
\text { gaufrée }\end{array}$ & ()bservations \\
\hline 1 & L'étirement et les constructions évoluent régulièrement et sans déformations. \\
\hline B & Les feuilles de cire se grondolent tris fortement et certains rayons sont tordus. \\
\hline C & $\begin{array}{l}\text { Les feuilles de cire se gondolent très jeu et les rayons construits sont très pet? } \\
\text { déformés. }\end{array}$ \\
\hline I) & $\begin{array}{l}\text { Les feuilles de cire se gondolent trés peu et les rayons construits sont trés pred } \\
\text { déformés. }\end{array}$ \\
\hline $\mathrm{E}$ & $\begin{array}{l}\text { Yombreuses construrtions parasites, paralleles an plan de la feuille de cire } \\
\text { gaufré ( } 1 \text { ). }\end{array}$ \\
\hline $\mathrm{I}$ & Létirement et les constructions évoluent régulièrement et sans déformations. \\
\hline
\end{tabular}

(r) Si les feuilles de cire gaufrée sont de taille insuffisante et ne couvrent pas tout le cadre, lo abillics édifient leurs cellules dans les partics libres d'abord.

$3^{\circ}$ Une trame métallique suffisante pour maintenir le fondement des bâtisses mais toutefois ne gênant pas la ponte de la reine.

\section{III. - Répartition régulière du couvain.}

Cette dernière qualité que nous avons retenue mérite également une attention particulière. Des cadres de couvain compacts et bien répartis sont évidemment très souhaitables pour l'obtention de colonies robustes. T,e tableau VI ci-joint résume les résultats de nos expériences en ce qui concerne l'aspect du couvain obtenu sur les différentes catégo- 
ries de cires gaufrées. I,es photographies aideront plus facilement à la compréhension des résultats qu'une longue discussion.

'TABI,EAL V'I

\begin{tabular}{|c|c|}
\hline latlegories de cire & Résultats ef observations \\
\hline A & (Couvain dense et regulier (repartition concentrigue). \\
\hline 13 & Couvain dense mais assey irrégulier. \\
\hline$i^{\circ}$ & 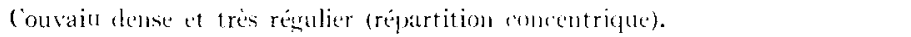 \\
\hline I) & Couvain dense matis un gen irrengulier. \\
\hline E & 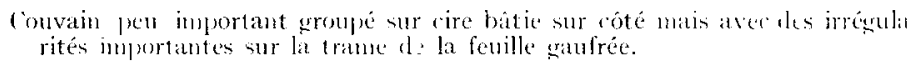 \\
\hline F & Couvain tries duse mais irregulier. \\
\hline
\end{tabular}

Deux catégories semblent satistaisantes : C et $A$.

En conclusion, il semble donc que parmi les six catégories de cires étudiées deux seulement sont près de réunir les trois qualités requises au début de cet exposé, les catégories $C$ et $A$. Un travail ultérieur nous permettra de fournir la formule d'me cire qui réponde d'une façon la plus parfaite possible aux trois exigences apicoles ci-dessus. I'ores et déjà la "cire pure d'abeilles" ne paraît pas indispensable et l'adjonction de cires minérales abaisse les prix sans nuire anx colonies. 